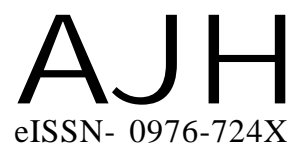

Article history :

Received : 06.06.2014

Revised : 18.09 .2014

Accepted : 04.10.2014
Members of the Research Forum

Associated Authors:

${ }^{1}$ Department of Vegetable Science, ACHF, Navsari Agriculture

University, NAVSARI (GUJARAT) INDIA

${ }^{2}$ ASPEE, Agricultural Research and Development Foundation, Malad (W), MUMBAI (M.S.) INDIA
Author for correspondence : P.B. JADHAV

ASPEE, Agricultural Research and Development Foundation, Malad (W), MUMBAI (M.S.) INDIA

Email :

tropicalsubtropical@gmail.com
THE ASIAN JOURNAL OF HORTICULTURE

Volume 9 | Issue 2 | Dec., 2014 |297-300

Visit us -www.researchjournal.co.in

\title{
Effect of foliar application of micronutrients in tomato (Lycopersicon esculentum Mill.) cv. GUJARAT TOMATO-2
}

\section{S.N. SARAVAIYA ${ }^{1}$, S.S. WAKCHAURE ${ }^{1}$, P.B. JADHAV, G.S. TEKALE ${ }^{1}$, N.B. PATIL $^{2}$ AND S.S. DEKHANE ${ }^{2}$}

ABSTRACT : The present investigation was undertaken with the main objective to study the effect of foliar application of micronutrients in tomato (Lycopersicon esculentum Mill.) cv. GUJARAT TOMATO-2 at ASPEE, ARDF, Tansa farm during Rabi season 2012-2013. The experiment consisted of eight treatments viz., $\mathrm{T}_{1}$ [RD NPK through chemical fertilizers $\left.\mathrm{N}: \mathrm{P}_{2} \mathrm{O}_{5}: \mathrm{K}_{2} \mathrm{O}_{5} \mathrm{~kg} \mathrm{ha}^{-1}(75: 37.5: 62.5)\right], \mathrm{T}_{2}$ $\left(\mathrm{T}_{1}+100 \mathrm{ppm} \mathrm{B}\right.$; i.e. boric acid $\left.0.571 \mathrm{~g} \mathrm{l}^{-1}\right), \mathrm{T}_{3}\left(\mathrm{~T}_{1}+100 \mathrm{ppm} \mathrm{Zn}\right.$; i.e. zinc sulphate $\left.0.246 \mathrm{~g} \mathrm{l}^{-1}\right), \mathrm{T}_{4}\left(\mathrm{~T}_{1}+\right.$ $100 \mathrm{ppm} \mathrm{Cu}$; i.e. copper sulphate $\left.0.420 \mathrm{~g} \mathrm{l}^{-1}\right), \mathrm{T}_{5}\left(\mathrm{~T}_{1}+100 \mathrm{ppm} \mathrm{Fe}\right.$; i.e. ferrous sulphate $\left.0.515 \mathrm{~g} \mathrm{l}^{-1}\right), \mathrm{T}_{6}$ $\left(\mathrm{T}_{1}+100 \mathrm{ppm} \mathrm{Mn}\right.$; i.e. manganese sulphate $\left.0.320 \mathrm{~g} \mathrm{l}^{-1}\right)$, and $\mathrm{T}_{7}\left(\mathrm{~T}_{1}+\right.$ mixture of all micronutrients $)$ and $\mathrm{T}_{8}\left(\mathrm{~T}_{1}+\right.$ multiplex $\left.4 \mathrm{ml} \mathrm{l}^{-1}\right)$ by mixing with simple water were imposed. The foliar application was made by using equipment knapsack sprayer in the evening hours. The thrice times foliar spray were made at 10 days interval starting from 40 days after transplanting seedling. The data clearly showed that the yield obtained with treatment $\mathrm{T}_{7}$ had significantly maximum plant height $(131.73 \mathrm{~cm})$, number of branches plant ${ }^{-1}(5.81)$, fresh weight of plants $\left(25.65 \mathrm{t} \mathrm{ha}^{-1}\right)$, dry matter yield of plants $(7670.03 \mathrm{~kg}$ ha $\left.^{-1}\right)$, maximum days to last picking (166.68), number of fruits plant ${ }^{-1}(34.26)$, fruit length $(5.52 \mathrm{~cm})$, fruit diameter $(4.64 \mathrm{~cm})$, fruit volume $\left(67.53 \mathrm{~cm}^{3}\right)$, single fruit weight $(49.20 \mathrm{~g})$, fruit weight plant ${ }^{-1}$ $(1.68 \mathrm{~kg})$, number of locules fruit ${ }^{-1}(3.03)$, pericarp thickness $(6.23 \mathrm{~mm})$, fruit yield ha ${ }^{-1}(46.78 \mathrm{t})$ and marketable fruit yield $\mathrm{ha}^{-1}(45.62 \mathrm{t})$. This treatment had maximum net return $(1,66,757 \mathrm{Rs} . / \mathrm{ha})$ and B:C Ratio $2.72: 1$ out all other treatments than over control.

KEY WORDS : Micronutrient, Tomato, GT-2

HOW TO CITE THIS ARTICLE : Saravaiya, S.N., Wakchaure, S.S., Jadhav, P.B., Tekale, G.S., Patil, N.B. and Dekhane, S.S. (2014). Effect of foliar application of micronutrients in tomato (Lycopersicon esculentum Mill.) cv. GUJARAT TOMATO-2. Asian J. Hort., 9(2): 297-300. 